\title{
Las comunidades del intermareal rocoso antes y después de la eliminación de un disturbio antropogénico: un caso de estudio en las costas de Puerto Madryn (Patagonia, Argentina)
}

\author{
The hard-bottom intertidal communities before and after removal of an anthropogenic disturbance: \\ A case study in the coast of Puerto Madryn (Patagonia, Argentina) \\ Américo Torres ${ }^{1}$ y Guillermo Caille ${ }^{2}$ \\ ${ }^{1}$ Centro Nacional Patagónico (CONICET), Brown 2915 (9120) Puerto Madryn, Chubut, Argentina \\ ${ }^{2}$ Fundación Patagonia Natural, Marcos A. Zar 670 (9120) Puerto Madryn, Chubut, Argentina \\ americo@cenpat.edu.ar
}

\begin{abstract}
In spite of the marked growth of the coastal cities in Patagonia, studies on the effects of the urban development upon marine intertidal communities are scarce. We evaluated the rocky-shore intertidal community composition during (June 1998) and after (June 2005) exposure to a sewage discharge which was eliminated in 2001. A perturbed environment and a control site were analyzed. No significant differences were
\end{abstract}

detected in species richness between sites and years. However, the species composition was different between sites in 1998 and similar in 2005. These results suggest the use of combined indicators in diagnostic studies on stressed environments.

Key words: Rocky shores, mid intertidal, environmental stress, sewage

\section{Introducción}

El vertido de aguas residuales e industriales es una de las principales razones del deterioro de los ecosistemas marinos costeros en todo el mundo (Cloern 2001). Estos líquidos poseen microorganismos patógenos, materia orgánica disuelta y particulada, sólidos suspendidos y elevados niveles de nutrientes inorgánicos que favorecen la eutroficación. En los últimos años, los disturbios ambientales relacionados a las descargas de aguas domésticas en ambientes costeros, han sido asociados al crecimiento de los asentamientos urbanos y a la ausencia de tratamientos adecuados (Cloern 2001). El uso de las comunidades bentónicas para valorar la contaminación marina y la calidad del agua, se basa en el concepto que éstas reflejan no sólo las condiciones al momento del muestreo, sino también aquellas a las cuales estuvieron expuestas (Pinedo et al. 2007).

Hasta el año 2001, la descarga de un efluente cloacal ( $\left.\sim 9000 \mathrm{~m}^{3} \mathrm{~d}^{-1}\right)$, proveniente de la planta de tratamiento secundario de la ciudad de Puerto Madryn ( $\sim 60 \mathrm{mil}$ habitantes), era el único ingreso de nutrientes de origen antrópico en Bahía Nueva. Debido a la baja eficiencia de la planta de tratamiento, las aguas estaban enriquecidas con nutrientes inorgánicos y materia orgánica disuelta (Gil et al. 2005). El objetivo de este estudio fue detectar las alteraciones en la estructura de una comunidad intermareal de sustrato rocoso, antes y después de cuatro años de la eliminación del efluente cloacal y estimar su capacidad de recuperación.

\section{Material y métodos}

La Bahía Nueva está localizada en el oeste del Golfo Nuevo (Fig. 1). En el intermareal de esta bahía se alternan playas de arena de pendiente suave, formaciones rocosas (restingas) de carbonatos y acantilados de unos $10 \mathrm{~m}$ de alto. La amplitud de marea es de 5,8 m, la circulación del agua es lenta $\left(<2,5 \mathrm{~cm} \mathrm{~s}^{-1}\right)$, y en dirección sur a norte (Rivas 1983). Este estudio fue realizado durante junio de 1998 y cuatro años después de la clausura de la descarga cloacal (junio de 2005) en el intermareal medio de la bahía (sitios 1 y 2) con sustrato rocoso y características similares en cuanto a grado de exposición al oleaje y al sol, pendiente ( $\sim 20^{\circ}$ grados), humedad y tamaño. El sitio 1 se ubicó a $10 \mathrm{~m}$ del punto de la descarga cloacal (Fig. 1) y el sitio 2 en Punta Cuevas (al sur del sitio 1), donde nunca existieron descargas de aguas residuales. Debido a la dirección predominante de las corrientes marinas, se asumió como nula la influencia cloacal sobre este último. Para determinar el número mínimo de muestras, se realizó un muestreo piloto en abril de 1998, utilizando como unidad muestral (UM) un cuadrado de 20 x $20 \mathrm{~cm}$.

En cada sitio se tomaron $10 \mathrm{UM}$ al azar, se reconocieron y contaron todos los ejemplares del macrobentos y se estimó, para ambos sitios, una curva del tipo $\mathrm{S}^{\prime}=\mathrm{a} \cdot \mathrm{N}^{\mathrm{b}}$, 


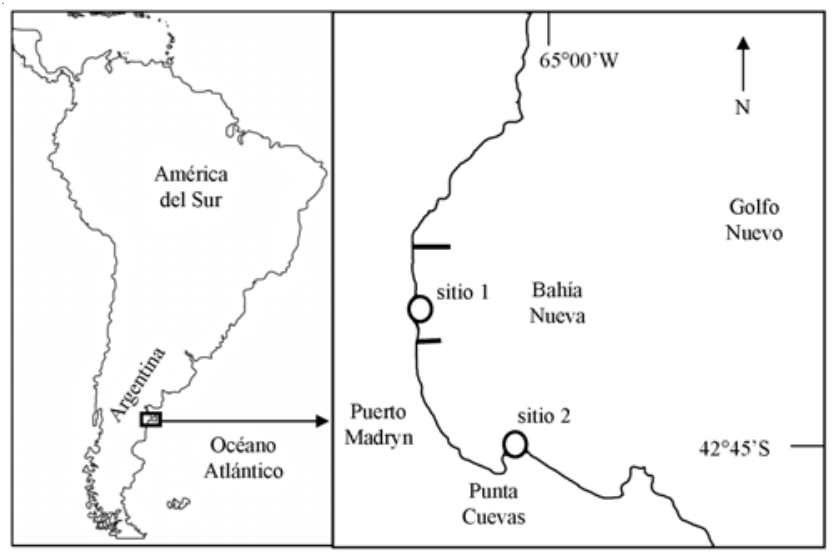

Figura 1

Ubicación de Bahía Nueva indicando la descarga del efluente (sitio 1) y el sitio control (sitio 2)

Location of Nueva bay indicating the discharge effluent outfall (site 1) and the control site (site 2)

\section{Tabla 1}

Frecuencia de ocurrencia (FO\%) para cada especie colectada en junio de 1998 y 2005 en cada sitio

Occurrence frequency (OF\%) for each species collected in June 1998 and 2005 in each site

\begin{tabular}{|c|c|c|c|c|}
\hline Especies & $\begin{array}{l}\text { Sitio 1 } \\
\text { (1998) }\end{array}$ & $\begin{array}{l}\text { Sitio 2 } \\
\text { (1998) }\end{array}$ & $\begin{array}{l}\text { Sitio } 1 \\
2005)\end{array}$ & $\begin{array}{l}\text { Sitio 2 } \\
(2005)\end{array}$ \\
\hline Brachidontes rodriguezi (D' orbigny, 1842) & 100 & 100 & 100 & 100 \\
\hline Ulva rigida (C. Agardh) Thuret & 100 & 100 & 100 & 100 \\
\hline Siphonaria lessoni (Blainville) & 100 & 100 & 100 & 100 \\
\hline Ralfsia verrucosa (Areschoug) & 100 & 100 & 90 & 90 \\
\hline Balanus glandula (Darwin, 1854) & 100 & 90 & 90 & 100 \\
\hline Corallina officinalis Linnaeus & 70 & 100 & 90 & 90 \\
\hline Anemonia chubutensis (Zamponi \& Acuña, 1992) & 10 & 100 & 60 & 20 \\
\hline Chaetomorpha sp. & 90 & 0 & 0 & 0 \\
\hline Ceramiun rubrum (Hudson) C. Agardh & 0 & 0 & 60 & 70 \\
\hline Sphacelaria cirrosa (Roth) C. Agardh & 0 & 0 & 40 & 0 \\
\hline Trophon geversianus (Pallas, 1974) & 10 & 80 & 10 & 30 \\
\hline Mytilus edulis platensis (D' orbigny, 1842) & 20 & 0 & 90 & 70 \\
\hline Tegula patagonica $\left(\mathrm{D}^{\prime}\right.$ orbigny, 1840$)$ & 0 & 90 & 0 & 0 \\
\hline Aulacomya atra atra (Molina, 1782) & 10 & 60 & 0 & 10 \\
\hline Polysiphonia argentinica Taylor & 0 & 70 & 20 & 70 \\
\hline Undaria pinnatifida (Harvey) Suringar & 0 & 0 & 20 & 30 \\
\hline Streblocladia camptoclada (Montagne) Falkenberg & 0 & 0 & 30 & 10 \\
\hline Diatomeas coloniales (no identificadas) & 50 & 10 & 0 & 0 \\
\hline Cladophora sp. & 60 & 0 & 0 & 0 \\
\hline Enteromorpha compressa (Linnaeus) Greville & 30 & 0 & 10 & 0 \\
\hline Polysiphonia sp. & 0 & 0 & 0 & 20 \\
\hline Dictyota dichotoma (Hudson) Lamouroux & 0 & 0 & 0 & 20 \\
\hline Tegula orbignyana (Pilsbry, 1900) & 0 & 0 & 0 & 20 \\
\hline Anotrichium furcellatum (J. Agardh) Baldock & 0 & 0 & 10 & 0 \\
\hline Cyrtograpsus angulatus (Dana, 1851) & 0 & 0 & 0 & 10 \\
\hline Isópodos (no identificados) & 10 & 0 & 0 & 0 \\
\hline
\end{tabular}


donde $\mathrm{N}$ es el número de UM y S' el número de especies encontradas (Valiela 1984, Slingsby \& Cook 1986). En ambos casos, un tamaño mínimo de 8 UM acumuló más del $90 \%$ del total de los taxones presentes, por lo cual se decidió tomar aleatoriamente 10 muestras de 40 x $40 \mathrm{~cm}$ en cada sitio. Todas las especies encontradas fueron contadas y determinadas taxonómicamente en laboratorio. La frecuencia de ocurrencia porcentual (FO\%) de cada especie fue calculada según: FO\% = 100 x (número de muestras presentes/número de muestras del sitio), y la riqueza de especies (S) (media \pm desviación estandar) fue estimada como el número de especies/muestra, registrada para cada sitio y en cada año muestreado. La prueba no paramétrica de Kruskal-Wallis (K-W) fue utilizada para evaluar las diferencias en la riqueza de especies entre sitios y años. Los análisis de agrupamientos, entre años, se realizaron por medio de un análisis multivariado del tipo UPGMA ('Unweighted Pair-Group Methods using Arithmetic Average', Sneath \& Sokal 1973), sobre las muestras tomadas en cada sitio. Con los taxones presentes en al menos dos muestras ( $\mathrm{FO} \%>10)$ se construyó una matriz del tipo presencia-ausencia (Hubalek 1982). Los taxones con $\mathrm{FO} \% \leq 10$, fueron considerados como 'poco frecuentes' y excluídos para no aportar incertidumbre en el análisis multivariado (Sneath \& Sokal 1973). Como medida de similitud se utilizó el índice de Jaccard (Hazel 1970), como medida de distorsión el coeficiente cofenético de correlación (CCC) (Sneath \& Sokal 1973) y se optó por el ligamiento promedio para construír los dendrogramas.

\section{Resultados y discusión}

En 1998, se hallaron 17 taxones en el sitio 1 y 12 en el sitio 2, mientras que en 2005 se hallaron 16 y 18 taxones respectivamente (Tabla 1 ). La riqueza de especies en 1998 fue $8,6 \pm 1,6$ especies/muestra para el sitio 1 y 9,9 $\pm 0,8$ para el sitio 2; mientras que en el 2005 fue 9,2 $\pm 2,5$ y 9,6 \pm 1,8 respectivamente. No se observaron diferencias significativas (K-W, $P>0,05)$ ni entre sitios ni entre años. El dendrograma para 1998 separó dos grupos de muestras, que se correspondieron con los sitios 1 y 2 y presentaron ensambles de especies diferentes (Fig. 2a). En la matriz de correlación (Tabla 2a) las especies presentes en ambos sitios, se separaron para formar tres grupos. El primero incluyó las siete especies con mayor FO\% (>50) y presentes en ambos sitios. El segundo incluyó cuatro especies con $\mathrm{FO} \%<50$, que estuvieron presentes sólo en el sitio 2, y que podrían ser consideradas de baja tolerancia. El tercer grupo incluyó tres especies con FO\% $<50$, que estuvieron presentes sólo en el sitio 1 , y serían favorecidas por el enriquecimiento orgánico (Díaz et al. 2002). El dendrograma obtenido en 2005, luego de cuatro años de eliminado el vertido, presentó un sólo agrupamiento de muestras, que contenía la mayoría de las especies (Fig. 2b). La matriz de correlación (Tabla 2b) presentó un sólo grupo principal, con las especies de mayor $\mathrm{FO} \%$ (>50) y que conforman la comunidad típica del intermareal medio sobre sustrato rocoso de la bahía.
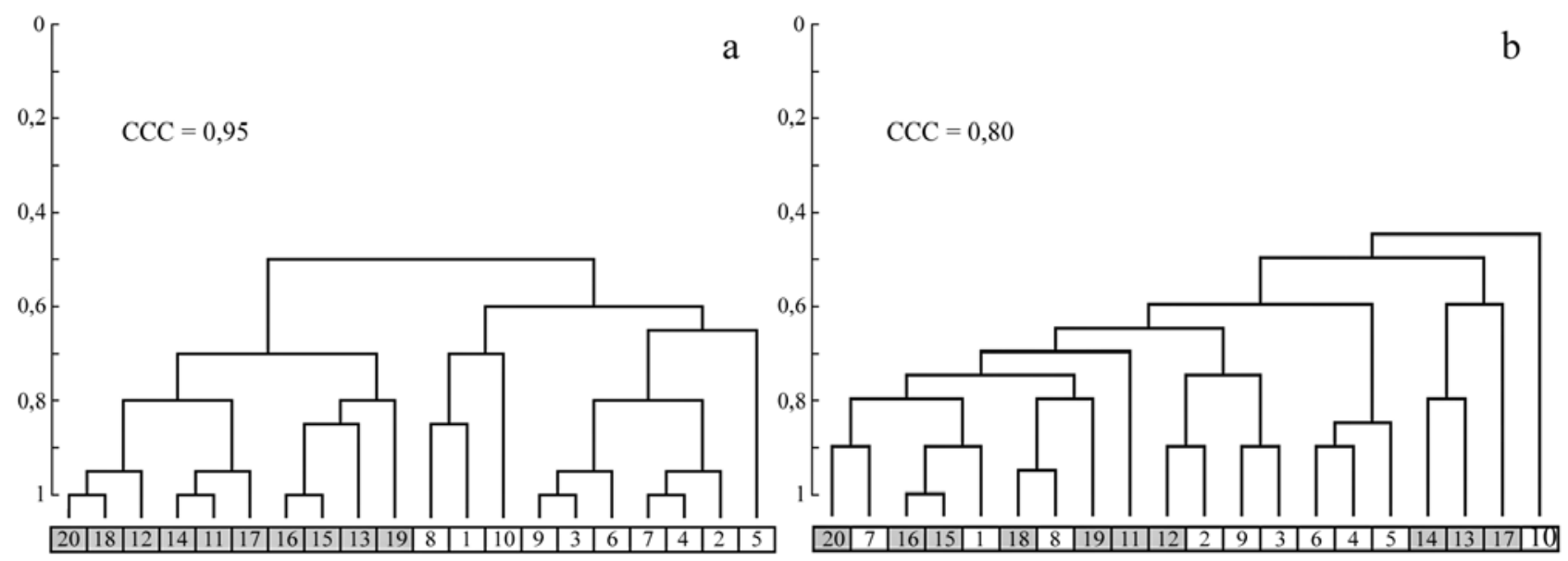

Figura 2

\author{
Dendrograma resultante del análisis entre muestras para 1998 (a) y 2005 (b). CCC: Coeficiente de \\ correlación cofenético. Sitio 1: muestras 1 a 10; sitio 2: muestras 11 a 20
}

Resultant dendrogram of analyses among samples for 1998 (a) and 2005 (b). CCC: Cophenetic correlation coefficient. Site 1: samples 1 to 10 ; site 2: samples 11 to 20 


\section{Tabla 2}

Distribución de especies acorde a los grupos reconocidos a un nivel de similitud de $50 \%$ en el análisis UPGMA para el año a) 1998 y b) 2005. Sitio 1: muestras 1 a 10; sitio 2: muestras 11 a 20; $x$ : especie presente en la muestra

Distribution of species according to groups recognized at a similarity level 50\% at level 50\% of similarity in UPGMA analysis for a) 1998 and b) 2005. Site 1: samples 1 to 10; site 2: samples 11

to 20; $\mathrm{x}$ : species present in the sample

\begin{tabular}{|c|c|c|c|c|c|c|c|c|c|c|c|c|c|c|c|c|c|c|c|c|}
\hline a & Especie/Muestra & 20 & 18 & 12 & 14 & 11 & 17 & 16 & 15 & 13 & 19 & 8 & 1 & 10 & 9 & 3 & 6 & 74 & 2 & 5 \\
\hline 14 & Cladophora sp. & & & & & & & & & & & & & & $\mathrm{x}$ & $\mathrm{x}$ & $\mathrm{x}$ & $x \quad x$ & $\mathrm{x}$ & \\
\hline 13 & Tube-dwelling diatoms & & & & & & & & & & & & & & $\mathrm{x}$ & $\mathrm{x}$ & & $x \quad x$ & $\mathrm{x}$ & \\
\hline 8 & Chaetomorpha sp. & & & & & & & & & $\mathrm{x}$ & & $\mathrm{x}$ & $\mathrm{x}$ & & $\mathrm{x}$ & $x$ & $\mathrm{x}$ & $x \quad x$ & $x$ & $\mathrm{x}$ \\
\hline 15 & Enteromorpha compressa & & & & & & & & & & & & & & $\mathrm{x}$ & $\mathrm{x}$ & $\mathrm{x}$ & & & \\
\hline 10 & Tegula patagonica & $\mathrm{x}$ & $\mathrm{x}$ & $\mathrm{x}$ & $\mathrm{x}$ & $\mathrm{x}$ & $\mathrm{x}$ & $\mathrm{x}$ & $\mathrm{x}$ & $\mathrm{x}$ & & & & & & & & & & \\
\hline 7 & Anemonia chubutensis & $\mathrm{x}$ & $\mathrm{x}$ & $\mathrm{x}$ & $\mathrm{x}$ & $\mathrm{x}$ & $\mathrm{x}$ & $\mathrm{x}$ & $\mathrm{x}$ & $\mathrm{x}$ & $\mathrm{x}$ & $\mathrm{x}$ & & & & & & & & \\
\hline 9 & Trophon geversiamus & $\mathrm{x}$ & $\mathrm{x}$ & $\mathrm{x}$ & $\mathrm{x}$ & $\mathrm{x}$ & $\mathrm{x}$ & & & $\mathrm{x}$ & $\mathrm{x}$ & & & & & & & & & $\mathrm{x}$ \\
\hline 12 & Polysiphonia argentinica & $\mathrm{x}$ & $\mathrm{x}$ & $\mathrm{x}$ & & & & $\mathrm{x}$ & $\mathrm{x}$ & $\mathrm{x}$ & $\mathrm{x}$ & & & & & & & & & \\
\hline 2 & Ulva rigida & $\mathrm{x}$ & $\mathrm{x}$ & $\mathrm{x}$ & $\mathrm{x}$ & $\mathrm{x}$ & $\mathrm{x}$ & $\mathrm{x}$ & $\mathrm{x}$ & $\mathrm{x}$ & $\mathrm{x}$ & $\mathrm{x}$ & $\mathrm{x}$ & $\mathrm{x}$ & $\mathrm{x}$ & $\mathrm{x}$ & $\mathrm{x}$ & $x \quad x$ & $\mathrm{x}$ & $\mathrm{x}$ \\
\hline 1 & Brachidontes rodriguezi & $\mathrm{x}$ & $\mathrm{x}$ & $\mathrm{x}$ & $\mathrm{x}$ & $\mathrm{x}$ & $\mathrm{x}$ & $\mathrm{x}$ & $\mathrm{x}$ & $\mathrm{x}$ & $\mathrm{x}$ & $\mathrm{x}$ & $\mathrm{x}$ & $\mathrm{x}$ & $\mathrm{x}$ & $\mathrm{x}$ & $\mathrm{x}$ & $x \quad x$ & $\mathrm{x}$ & $\mathrm{x}$ \\
\hline 3 & Siphonaria lessoni & $\mathrm{x}$ & $\mathrm{x}$ & $\mathrm{x}$ & $\mathrm{x}$ & $\mathrm{x}$ & $\mathrm{x}$ & $\mathrm{x}$ & $\mathrm{x}$ & $\mathrm{x}$ & $\mathrm{x}$ & $\mathrm{x}$ & $\mathrm{x}$ & $\mathrm{x}$ & $\mathrm{x}$ & $\mathrm{x}$ & $x \quad x$ & $x \quad x$ & $\mathrm{x}$ & $\mathrm{x}$ \\
\hline 4 & Ralfsia verrucosa & $x$ & $\mathrm{x}$ & $\mathrm{x}$ & $x$ & $x$ & $x$ & $x$ & $x$ & $x$ & $x$ & & $x$ & $x$ & $x$ & $x$ & $x$ & $\begin{array}{ll}x & x\end{array}$ & $x$ & $\mathrm{x}$ \\
\hline 5 & Balamus glandula & $\mathrm{X}$ & $\mathrm{x}$ & & $\mathrm{x}$ & $\mathrm{x}$ & $\mathrm{x}$ & $\mathrm{x}$ & $\mathrm{x}$ & $\mathrm{x}$ & $\mathrm{x}$ & $\mathrm{x}$ & $\mathrm{x}$ & $\mathrm{x}$ & $\mathrm{x}$ & $\mathrm{x}$ & $\mathrm{x}$ & $x \quad x$ & $\mathrm{x}$ & $\mathrm{x}$ \\
\hline 6 & Corallina officinalis & $\mathrm{x}$ & $\mathrm{x}$ & $\mathrm{x}$ & $\mathrm{x}$ & $\mathrm{x}$ & $\mathrm{x}$ & $\mathrm{x}$ & $\mathrm{x}$ & $\mathrm{x}$ & $\mathrm{x}$ & & & & $\mathrm{x}$ & $\mathrm{x}$ & $\mathrm{x}$ & $x \quad x$ & $\mathrm{x}$ & $\mathrm{x}$ \\
\hline 11 & Aulacomya atra atra & & & & $\mathrm{x}$ & $\mathrm{x}$ & & $\mathrm{x}$ & $\mathrm{x}$ & $\mathrm{x}$ & $\mathrm{x}$ & & & & & & & & $\mathrm{x}$ & \\
\hline b & Especie/Muestra & 20 & 7 & 16 & 15 & 1 & 18 & 8 & 19 & 11 & 122 & 29 & 3 & 6 & 4 & 5 & 14 & 13 & 17 & 10 \\
\hline 14 & Sphacelaria cirrosa & & & & & $\mathrm{x}$ & & & & & & & & $\mathrm{x}$ & $\mathrm{x}$ & $\mathrm{x}$ & & & & \\
\hline 12 & Streblocladia camptoclada & & & & & & & $\mathrm{x}$ & & & & & & $\mathrm{x}$ & & $\mathrm{x}$ & & $\mathrm{x}$ & & \\
\hline 13 & Trophon geversiamus & & & & & & $\mathrm{x}$ & $\mathrm{x}$ & & & & & & & & & $\mathrm{x}$ & $\mathrm{x}$ & & \\
\hline 9 & Polysiphonia argentinica & & & & & & $\mathrm{x}$ & $\mathrm{x}$ & $\mathrm{x}$ & $\mathrm{x}$ & $\mathrm{x}$ & $x$ & & & & & $\mathrm{x}$ & $\mathrm{x}$ & $\mathrm{x}$ & \\
\hline 6 & Ralfsia verrucosa & $\mathrm{x}$ & $\mathrm{x}$ & $x$ & $x$ & $x$ & $\mathrm{x}$ & $\mathrm{x}$ & $\mathrm{x}$ & $\mathrm{x}$ & $\mathrm{x}$ & $\mathrm{x}$ & $x$ & $x$ & $\mathrm{x}$ & $\mathrm{x}$ & $\mathrm{x}$ & $\mathrm{x}$ & & \\
\hline 5 & Corallina officinalis & $\mathrm{x}$ & $\mathrm{x}$ & $\mathrm{x}$ & $\mathrm{x}$ & $\mathrm{x}$ & $\mathrm{x}$ & $\mathrm{x}$ & $\mathrm{x}$ & & $\mathrm{x}$ & $\mathrm{x}$ & $x$ & $x$ & $\mathrm{x}$ & $\mathrm{x}$ & $\mathrm{x}$ & $\mathrm{x}$ & $\mathrm{x}$ & \\
\hline 2 & Brachidontes rodriguezi & $\mathrm{x}$ & $\mathrm{x}$ & $\mathrm{x}$ & $\mathrm{x}$ & $\mathrm{x}$ & $\mathrm{x}$ & $\mathrm{x}$ & $\mathrm{x}$ & $\mathrm{x}$ & $\mathrm{x}$ & $\mathrm{x}$ & $x$ & $x$ & $\mathrm{x}$ & $\mathrm{x}$ & $\mathrm{x}$ & $\mathrm{x}$ & $\mathrm{x}$ & $\mathrm{x}$ \\
\hline 1 & Ulva rigida & $\mathrm{x}$ & $\mathrm{x}$ & $\mathrm{x}$ & $\mathrm{x}$ & $\mathrm{x}$ & $\mathrm{x}$ & $\mathrm{x}$ & $\mathrm{x}$ & $\mathrm{x}$ & $\mathrm{x}$ & $\mathrm{x}$ & $x$ & $x$ & $\mathrm{x}$ & $\mathrm{x}$ & $\mathrm{x}$ & $\mathrm{x}$ & $\mathrm{x}$ & $\mathrm{x}$ \\
\hline 3 & Siphonaria lessoni & $\mathrm{x}$ & $\mathrm{x}$ & $\mathrm{x}$ & $\mathrm{x}$ & $\mathrm{x}$ & $\mathrm{x}$ & $\mathrm{x}$ & $\mathrm{x}$ & $\mathrm{x}$ & $\mathrm{x}$ & $\mathrm{x}$ & $x$ & $x$ & $\mathrm{x}$ & $\mathrm{x}$ & $\mathrm{x}$ & $\mathrm{x}$ & $\mathrm{x}$ & $\mathrm{x}$ \\
\hline 4 & Balamus glandula & $\mathrm{x}$ & $\mathrm{x}$ & $\mathrm{x}$ & $\mathrm{x}$ & $\mathrm{x}$ & $\mathrm{x}$ & $\mathrm{x}$ & $\mathrm{x}$ & $\mathrm{x}$ & $\mathrm{x}$ & $\mathrm{x}$ & $x$ & $x$ & $\mathrm{x}$ & $\mathrm{x}$ & $\mathrm{x}$ & $\mathrm{x}$ & $\mathrm{x}$ & $\mathrm{x}$ \\
\hline 7 & Mytilus edulis platensis & $\mathrm{x}$ & $\mathrm{x}$ & $\mathrm{x}$ & $\mathrm{x}$ & $\mathrm{x}$ & $\mathrm{x}$ & $\mathrm{x}$ & $\mathrm{x}$ & $\mathrm{x}$ & $\mathrm{x}$ & $x \quad x$ & $x$ & $x$ & $\mathrm{x}$ & $\mathrm{x}$ & & & & \\
\hline 8 & Ceramiun rubrum & $\mathrm{x}$ & $\mathrm{x}$ & $\mathrm{x}$ & $\mathrm{x}$ & $\mathrm{x}$ & $\mathrm{x}$ & $\mathrm{x}$ & $\mathrm{x}$ & $\mathrm{x}$ & & & & $\mathrm{x}$ & $\mathrm{x}$ & & & $\mathrm{x}$ & & $\mathrm{x}$ \\
\hline 10 & Anemonia chubutensis & & $\mathrm{x}$ & & & & $\mathrm{x}$ & $\mathrm{x}$ & $\mathrm{x}$ & & & & $\mathrm{x}$ & $x$ & $\mathrm{x}$ & $\mathrm{x}$ & & & & \\
\hline 11 & Undaria pinnatifida & $\mathrm{x}$ & $\mathrm{x}$ & & & & $\mathrm{x}$ & $\mathrm{x}$ & & & & & & & & & & & $\mathrm{x}$ & \\
\hline
\end{tabular}

Los resultados obtenidos señalan que los sitios perturbados (como el sitio 1) se caracterizarían tanto por la ausencia de algunas especies (Polysiphonia argentinica), como por la presencia de otras (Cladophora sp., Enteromorpha compressa y Chaetomorpha sp.). Teniendo en cuenta que el número de muestras tomadas es bajo, este debería aumentar para dar mayor robustez estadística al estudio y confirmar los resultados obtenidos. Además, consideramos adecuado aumentar el número de sitios control, para descartar que las diferencias halladas puedan ser específicas de cada sitio y no necesariamente sean debidas a la descarga del efluente. Sin embargo, por su sencilla aplicación, nuestros ensayos señalan la utilidad de la comparación entre sitios impactados y sitios control, y del uso de indicadores combinados de estrés en estos ambientes, para orientar estudios de mayor complejidad. El caso presentado resalta también la importancia de los estudios a largo plazo en ambientes marinos costeros perturbados, tanto para evaluar su capacidad de cambio frente a disturbios antrópicos, como para proponer los principales lineamientos para elaborar las pautas de manejo tendientes a su recuperación. 


\section{Agradecimientos}

A la Dra. Graciela Casas (CENPAT), por su ayuda en los análisis taxonómicos. Este estudio ha sido financiado parcialmente por el Proyecto ARG/02/G31, GEF- PNUD. Agradecemos a los revisores anónimos y editor por sus valiosos comentarios para mejorar la estructura del trabajo.

\section{Literatura citada}

Cloern JE. 2001. Our evolving conceptual model of the coastal eutrophication problem. Marine Pollution Bulletin 210: 223-253.

Diaz P, JJ López-Gappa \& M Piriz. 2002. Symptoms of eutrophication in intertidal macroalgal assemblages of Nuevo Gulf (Patagonia, Argentina). Botanica Marina 45: 267-273.

Gil MN, A Torres \& JL Esteves. 2005. Uptake of sewage derived nitrogen by Ulva rigida (Chlorophyceae) in Bahía Nueva (Golfo Nuevo, Patagonia, Argentine). Hydrobiologia 532: $39-43$
Hazel JE. 1970. Binary coefficients and clustering in bioestratigraphy. Bulletin of the Geological Society of America 81: 3237-3252.

Hubalek Z. 1982. Coefficients of associations and similarity based on binary (presence-absence) data: an evaluation. Biological Reviews 57: 669-689.

Pinedo S, M Carcía, MP Satta, M De Torres \& E Ballesteros. 2007. Rocky-shore communities as indicators of water quality: a case study in the northwestern mediterranean. Marine Pollution Bulletin 55: 126-135.

Rivas AL. 1983. Análisis de la circulación costera en el Golfo Nuevo. Acta Oceanographica Argentina 3(2): 49-66.

Slingsby D \& C Cook. 1986. Practical ecology, 213 pp. MacMillan, Londres.

Sneath PHA \& RR Sokal. 1973. Numerical taxonomy: the principles and practice of numerical classification, $573 \mathrm{pp}$. Freeman \& Company, San Francisco.

Valiela I. 1984. Marine ecological processes, 546 pp. Springer Verlag, New York.

Recibido el 16 de septiembre de 2008 y aceptado el 20 de marzo de 2009 